\title{
Role of leptin in preeclampsia
}

\section{Reshma Devarajachar ${ }^{1}$, Harish Rangareddy ${ }^{2 *}$}

\author{
${ }^{1}$ Department of Biochemistry, Sapthagiri Institute of Medical Sciences and Research Center, Bengaluru, Karnataka, \\ India \\ ${ }^{2}$ Department of Biochemistry, Sri Devaraj Urs Medical College, constituent college of Sri Devaraj Urs Academy of \\ Higher Education, Kolar, Karnataka, India
}

Received: 09 December 2020

Accepted: 13 January 2021

\author{
*Correspondence: \\ Dr. Harish Rangareddy, \\ E-mail: harishreddy1349@gmail.com
}

Copyright: (C) the author(s), publisher and licensee Medip Academy. This is an open-access article distributed under the terms of the Creative Commons Attribution Non-Commercial License, which permits unrestricted non-commercial

\begin{abstract}
Preeclampsia is one of the common complications of pregnancy and associated with elevated leptin concentrations. In this review the potential sources of leptin are explored and the factors that contribute to maternal increased concentrations of leptin include obesity, insulin resistance and hypoxia. Further research is warranted to understand the role of leptin in pathogenesis of preeclampsia.
\end{abstract}

Keywords: Leptin, Preeclampsia

\section{INTRODUCTION}

Preeclampsia is a hypertensive disorder complicating pregnancy and an important cause for both maternal and fetal morbidity and mortality in developing countries. The prevalence of preeclampsia is $2-8 \%$ of all pregnancies worldwide. ${ }^{1}$ Preeclampsia is characterized by maternal hypertension, edema and proteinuria. These symptoms develop after 20 weeks of gestation and disappear within 7-10 days post partum. The pathogenesis of preeclampsia is still not completely understood and is explained based on the mechanisms involving endothelial dysfunction, inflammation and oxidative stress. ${ }^{2}$ Preeclampsia can complicate pregnancy leading to eclampsia that is the onset of convulsions in woman with preeclampsia which cannot be attributed to any other cause. There are two stages of preeclampsia; first stage of hypoxia due to reduced placental perfusion caused by defective trophoblastic invasion and second stage of maternal systemic manifestations constituting of inflammatory response, metabolic and vascular dysfunction which can lead to multiorgan dysfunction. ${ }^{1}$

Leptin is a $16 \mathrm{kDa}$ protein neuroendocrine hormone that plays an important role in energy homeostasis, reproductive biology and as a satiety factor. It is expressed abundantly and specifically in the adipose tissue. Leptin decreases appetite mediating via its cognate receptor (ObR) in the hypothalamus. The other effects include activation sympathetic nervous system and increases energy expenditure. Thus, leptin has a role in energy metabolism and decreases body weight and adiposity. ${ }^{3}$ In pregnant women there are drastic changes in energy metabolism which is well documented. The energy requirements of the fetus are met through increased food intake, decreased insulin sensitivity and hyperlipidemia. Placental hormones, prolactin and steroid hormones are implied to play a major role in maternal adaptation to the pregnant state. ${ }^{4}$

\section{BIOCHEMICAL FUNCTIONS OF LEPTIN}

Leptin production occurs in placenta during pregnancy while initially it was thought to be exclusively produced by white adipose tissue. The receptor for leptin is expressed in various maternal tissues, placenta and fetal tissues abundantly. Hence, the role of leptin in physiology of pregnancy and pathophysiology of preeclampsia is anticipated. The production and metabolism of leptin during pregnancy are significantly different among 
species. ${ }^{5}$ Subsequent to the discovery of genes for leptin and it's receptor, experimental animal studies and in vitro studies the various functions of leptin have been realized. Apart from the effect on satiety and activation of sympathetic nervous system, leptin affects other biochemical functions such as carbohydrate metabolism, lipid metabolism, insulin sensitivity, hematopoiesis, angiogenesis, blood pressure and gonadal hormone secretion in the ovary. Leptin plays a pivotal role in reproduction by acting as a metabolic signal neuroendocrine and reproductive system. ${ }^{6}$

In humans, there have been numerous studies which demonstrated that maternal peripheral leptin levels are increased during pregnancy. It has also been hypothesized that leptin production is in turn modulated by the reproductive hormones. Estrogen has been suggested to stimulate leptin production and it has been observed that throughout the menstrual cycle leptin and estradiol have been reported to have similar profiles. ${ }^{7}$

There are overlapping features between obesity and preeclampsia such as glucose intolerance, insulin resistance, hyperlipidemia and hypertension. Maternal obesity predisposes to preeclampsia and several studies have focused on the relationship between leptin secreted from the adipose tissue and preeclampsia. Plasma leptin levels in subjects who are not pregnant is directly related to the body mass index (BMI) and it is observed that leptin concentrations increase in subjects with insulin resistance. In uncomplicated pregnancies, a two to four fold increase in leptin levels has been observed, peaking in second trimester and declining post partum. ${ }^{8}$ It has been observed in women suffering from miscarriage have low serum leptin concentrations. ${ }^{9}$ Though the mechanism of gestational increase of leptin is yet to be explored this indicates that leptin does play a crucial role in the outcome of pregnancy.

\section{SOURCES OF LEPTIN}

Human placenta is now recognized to be a source of leptin with the mRNA required for the translation and the protein colocalized with leptin receptors in the syncitiotrophoblast. The human placental leptin gene also has a specific promoter region, which may suggest that placental leptin may be differentially regulated compared with leptin of adipose origin. The size of the placenta has been reported to correlate with the maternal and cord concentrations of the leptin implying that placenta may not be just the source of the hormone but also a site for the action of leptin. ${ }^{10}$

Obesity is a risk factor for preeclampsia and preeclampsia is associated with insulin resistance, dyslipidemia especially elevation of fatty acids and triglycerides. The placenta also has an undisputed role in the origin of preeclampsia and alterations of circulating leptin may form the crux of the pathogenesis of preeclampsia. ${ }^{11}$
The two evident potential sources of maternal blood leptin concentrations are maternal adiposity and placenta. In nonpregnant subjects, the leptin levels and adiposity are related with the obvious correlation with the BMI. During pregnancy the BMI not only reflects the adiposity but also the variable contributions from the products of conception and volume expansion. Hence, there has been evaluation of maternal blood concentrations of leptin and the BMI before pregnancy by some investigators. ${ }^{12}$

Insulin resistance is frequent in women with preeclampsia and increased concentrations of leptin are associated with insulin resistance in non-pregnant subjects. ${ }^{12}$ Corollary of this insulin resistance may be the maternal increase of leptin during pregnancy. There is evidence that there is no association between leptin concentration and insulin resistance as assessed by an oral glucose test in women with preeclampsia. ${ }^{13}$ However, it was observed that maternal insulin concentrations correlated with plasma leptin in both normotensive and preeclamptic pregnancies. This could imply that insulin may be a stimulus for the synthesis of leptin during pregnancy independent of preeclampsia. ${ }^{14}$ The increase in adiposity or insulin resistance would not suffice as the explanation for elevated maternal leptin levels in preeclampsia and the placenta is an obvious alternative source. Northern blot analysis has shown that the leptin mRNA levels are increased in placentas of preeclamptic women compared with normotensive pregnancies. ${ }^{15}$ It has also been observed that maternal leptin concentrations decreased post partum to levels anticipated for the women's BMI.

The leptin gene expression is linked to a fall in neuropeptide Y (NPY) expression. An experimental study has shown that leptin suppresses the expression of NPY in mouse hypothalamus. NPY is a potent vasoconstriction and it may be hypothesized that suppression of its expression in the placenta could be of benefit as the main etiology in preeclampsia is vasoconstriction. ${ }^{16}$ However, a simple relationship between placental mass and leptin synthesis is an unlikely explanation as placental mass is generally reduced in preeclampsia, particularly if the disease is severe and of early onset. Hypoxia may be another contributing factor as evidenced from the observations that women at risk of preeclampsia often demonstrate abnormalities of the uterine artery Doppler flow velocity waveform. Findings of uterine artery Doppler are indicative of poor placentation and partial failure of trophoblast invasion. ${ }^{17}$ This suggests that the hypoperfused placenta may be hypoxic leading to the failure of placental growth. It is now recognized that leptin is a proinflammatory adipocytes derived factor. ${ }^{18}$

The leptin levels are increased in maternal circulation as a response to hypoxic conditions and can be considered a marker of placental ischemia. This feedback mechanism could possible result increased nutrient delivery to the underperfused placenta. ${ }^{19}$ 


\section{OXIDATIVE STRESS AND PREECLAMPSIA}

The endothelial dysfunction in preeclampsia has been attributed to the increased oxidative stress as witnessed with the increased Malondialdehyde (MDA) levels. MDA, a metabolite of lipid peroxidation which is enhanced in preeclampsia is implicated to be associated with decreased placental perfusion. $^{20}$ Placental oxidative stress and maternal inflammatory responses are immensely increased in preeclampsia. The defective trophoblastic proliferation may tip off the balance between the lipid peroxides like MDA and antioxidant systems leading to endothelial dysfunction. Furthermore the hypoxic condition may facilitate the production of free radicals from the placenta that cause lipid peroxidation. ${ }^{21}$ This placental oxidative stress may lead to lead to increased production and maternal circulation of factors that stimulate the inflammatory responses and activate the maternal endothelial cells like various cytokines comprising of interleukins (IL-6, 8, 10) and tumor necrosis factor - $\alpha$ (TNF $-\alpha$ ) and the novel proinflammatory adipocyte derived cytokine leptin. ${ }^{2}$ Pregnancy is a phase of increased nutritional requirements which is responsible for the cell differentiation growth of the fetus. Micronutrients have an important influence on the health of the pregnant women and growing fetus and these have to be supplemented as a daily requirement through diet. ${ }^{22}$ Deficiency of some trace elements make pregnant women vulnerable for developing preeclampsia as these regulate the balance between free radicals and antioxidants. Alterations in the levels of essential trace elements zinc and copper have been implicated in the development of preeclampsia. ${ }^{23}$ These factors could contribute to the increased oxidative stress and pathogenesis of preeclampsia.

\section{CONCLUSION}

Maternal leptin concentrations appear to be increased in preeclampsia. This review addresses the role of leptin in preeclampsia and its possible protective effect by mediating the suppression of NPY, increasing the perfusion of the placenta and placental size. The raise in inflammatory markers in preeclampsia, lipid peroxidation and alterations in trace elements creates a scope for further investigation of leptin and oxidative stress markers in clinic and laboratory.

\section{Funding: No funding sources \\ Conflict of interest: None declared \\ Ethical approval: Not required}

\section{REFERENCES}

1. Jeyabalan A. Epidemiology of preeclampsia: impact of obesity. Nutr Rev. 2013;71:S18-S25.

2. Sharma A, Satyam A, Sharma JB. Leptin, IL-10 and Inflammatory Markers (TNF- $\alpha$, IL-6 and IL-8) in PreEclamptic, Normotensive Pregnant and Healthy NonPregnant Women. American Journal of Reproductive Immunology. 2007;58:21-30.
3. Ramos-Lobo AM, Donato J Jr. The role of leptin in health and disease. Temperature (Austin). 2017;4(3):258-291.

4. Napso T, Yong HEJ, Lopez-Tello J, Sferruzzi-Perri AN. The Role of Placental Hormones in Mediating Maternal Adaptations to Support Pregnancy and Lactation. Front Physiol. 2018;9:1091.

5. Pérez-Pérez A, Toro A, Vilariño-García T, Maymó J, Guadix P, Dueñas JL et al. Leptin action in normal and pathological pregnancies. J Cell Mol Med. 2018;22(2):716-727.

6. Kelesidis T, Kelesidis I, Chou S, Mantzoros CS. Narrative review: the role of leptin in human physiology: emerging clinical applications. Ann Intern Med. 2010;152(2):93-100.

7. Ajala OM, Ogunro PS, Elusanmi GF, Ogunyemi OE, Bolarinde AA. Changes in serum leptin during phases of menstrual cycle of fertile women: relationship to age groups and fertility. Int $\mathrm{J}$ Endocrinol Metab. 2013;11(1):27-33.

8. Sattar N, Greer IA, Pirwani I, Gibson J, Wallace AM. Leptin levels in pregnancy: marker for fat accumulation and mobilization? Acta Obstet Gynecol Scand. 1998;77(3):278-83.

9. Laird SM, Quinton ND, Anstie B, Li TC, Blakemore AI. Leptin and leptin-binding activity in women with recurrent miscarriage: correlation with pregnancy outcome. Hum Reprod. 2001;16(9):2008-13.

10. Challier J, Galtier M, Bintein T, Cortez A, Lepercq J, Hauguel-de Mouzon S. Placental leptin receptor isoforms in normal and pathological pregnancies. Placenta. 2003;24(1):92-9.

11. Lopez-Jaramillo P, Barajas J, Rueda-Quijano SM, Lopez-Lopez C, Felix C. Obesity and Preeclampsia: Common Pathophysiological Mechanisms. Front Physiol. 2018;9:1838.

12. Thagaard IN, Hedley PL, Holm JC, Lange T, Larsen $\mathrm{T}$, Krebs $\mathrm{L}$ and Christiansen $\mathrm{M}$ : Leptin and adiponectin as markers for preeclampsia in obese pregnant women, a cohort study. Pregnancy Hypertens. 2019;15:78-83.

13. Xiao WQ, He JR, Shen SY, Lu JH, Kuang YS, Wei $\mathrm{XL}$ et al. Maternal circulating leptin profile during pregnancy and gestational diabetes mellitus. Diabetes Research and Clinical Practice. 2020;161:108041.

14. Taylor BD, Ness RB, Olsen J, Hougaard DM, Skogstrand K, Roberts JM et al. Serum leptin measured in early pregnancy is higher in women with preeclampsia compared with normotensive pregnant women. Hypertension. 2015;65(3):594-9.

15. Mise H, Sagawa N, Matsumoto T, Yura S, Nanno H, Itoh $\mathrm{H}$ et al. Augmented placental production of leptin in preeclampsia: possible involvement of placental hypoxia. J Clin Endocrinol Metab. 1998;83(9):32259.

16. Dotsch J, Nusken KD, Knerr I, Kirschbaum M, Repp $\mathrm{R}$, Rascher W. Leptin and neuropeptide $\mathrm{Y}$ gene expression in human placenta: ontogeny and evidence for similarities to hypothalamic regulation. The 
Journal of Clinical Endocrinology \& Metabolism. 1999;84(8):2755-8.

17. Giordano R, Cacciatore A, Romano M, La Rosa B, Fonti I, Vigna R. Uterine artery Doppler flow studies in obstetric practice. J Prenat Med. 2010;4(4):59-62.

18. Salimi S, Farajian-Mashhadi F, Naghavi A, Mokhtari M, Shahrakipour M, Saravani $M$ et al. Different profile of serum leptin between early onset and late onset preeclampsia. Disease markers. 2014;2014.

19. Laivuori H, Gallaher MJ, Collura L, Crombleholme WR, Markovic N, Rajakumar A et al. Relationships between maternal plasma leptin, placental leptin mrna and protein in normal pregnancy, pre-eclampsia and intrauterine growth restriction without pre-eclampsia. Mol Hum Reprod. 2006;12:551-556.

20. Bakacak M, Kılınç M, Serin S, Ercan Ö, Köstü B, Avc1 F, et al. Changes in Copper, Zinc, and Malondialdehyde Levels and Superoxide Dismutase Activities in Pre-Eclamptic Pregnancies. Medical science monitor: international medical journal of experimental and clinical research. 2015;21:2414.

21. Redman CW, Sargent IL. Placental stress and preeclampsia: a revised view. Placenta. 2009;30(SupplA):S38-42.

22. Black RE. Micronutrients in pregnancy. Br J Nutr. 2001;85:93-197.

23. Rathore S, Gupta A, Batra HS, Rathore R. Comparative study of trace elements and serum ceruloplasmin level in normal and pre-eclamptic pregnancies with their cord blood. Biomed Res. 2011;22:207-10.

Cite this article as: Devarajachar R, Rangareddy $\mathrm{H}$. Role of leptin in preeclampsia. Int J Reprod Contracept Obstet Gynecol 2021;10:814-7. 